\title{
Starspots on WASP-107 and pulsations of WASP-118
}

\author{
T. Močnik, ${ }^{\star}$ C. Hellier, D. R. Anderson, B. J. M. Clark and J. Southworth \\ Astrophysics Group, Keele University, Staffordshire, ST5 5BG, UK
}

Accepted 2017 April 20. Received 2017 April 12; in original form 2017 February 17

\begin{abstract}
By analysing the $K 2$ short-cadence photometry, we detect starspot occultation events in the light curve of WASP-107, the host star of a warm-Saturn exoplanet. WASP-107 also shows a rotational modulation with a period of $17.5 \pm 1.4 \mathrm{~d}$. Given that the rotational period is nearly three times the planet's orbital period, one would expect in an aligned system to see starspot occultation events to recur every three transits. The absence of such occultation recurrences suggests a misaligned orbit unless the starspots' lifetimes are shorter than the star's rotational period. We also find stellar variability resembling $\gamma$ Doradus pulsations in the light curve of WASP-118, which hosts an inflated hot Jupiter. The variability is multiperiodic with a variable semi-amplitude of $\sim 200 \mathrm{ppm}$. In addition to these findings, we use the $K 2$ data to refine the parameters of both systems and report non-detections of transit-timing variations, secondary eclipses and any additional transiting planets. We used the upper limits on the secondary-eclipse depths to estimate upper limits on the planetary geometric albedos of 0.7 for WASP-107b and 0.2 for WASP-118b.
\end{abstract}

Key words: stars: individual: (WASP-107, WASP-118) - stars: oscillations - planetary systems - starspots.

\section{INTRODUCTION}

The Kepler (Borucki et al. 2010) and K2 (Howell et al. 2014) missions have provided the community with high-precision photometric observations of 2449 confirmed transiting exoplanets to date among a total of 2716 confirmed transiting exoplanets, according to NASA Exoplanet Archive. ${ }^{1}$ In addition, $K 2$ is also observing exoplanets previously found by the ground-based transit surveys such as WASP (Pollacco et al. 2006). WASP-107 and WASP-118 are among the brightest systems observed by $K 2$, which allows for detailed characterization owing to high-precision light curves combined with existing spectroscopic observations performed by Anderson et al. (2017) and Hay et al. (2016).

WASP-107b is a warm Saturn in a 5.7-d orbit around a $V=11.6$, K6 main-sequence star (Anderson et al. 2017). The planet lies in the transition region between ice giants and gas giants, with a mass of $2.2 M_{\text {Nep }}$ or $0.12 M_{\text {Jup }}$, but an inflated radius of $0.94 R_{\text {Jup }}$. The WASP discovery photometry revealed a possible stellar rotational modulation with a period of $\sim 17 \mathrm{~d}$ and an amplitude of 0.4 per cent. This led Anderson et al. (2017) to propose that the host star is magnetically active.

WASP-118b is an inflated hot Jupiter with a mass of $0.51 M_{\text {Jup }}$ and a radius of $1.4 R_{\text {Jup }}$ It orbits a $V=11.0 \mathrm{~F} 6 \mathrm{IV} / \mathrm{V}$ star every $4.0 \mathrm{~d}$ (Hay et al. 2016).

\footnotetext{
^E-mail: t.mocnik@keele.ac.uk

${ }^{1} \mathrm{http}: / /$ exoplanetarchive.ipac.caltech.edu/
}

If a transiting planet crosses a starspot, it will produce a temporary brightening in the transit (Silva 2003). Starspot occultation events can provide an accurate measurement of the stellar rotational period and the obliquity, i.e. the angle between the stellar rotational axis and the planet's orbital axis. Obliquities can tell us about a planet's dynamical history and about planet migration mechanisms. If the obliquity is small, the same starspot can be occulted recurrently at different stellar longitudes, such as in the case of an aligned Qatar-2 system (Močnik, Southworth \& Hellier 2016a; Dai et al. 2017). Alternatively, if a system is misaligned, the transit chord will cross stellar active latitudes only at certain preferential phases, such as in the case of HAT-P-11b (Sanchis-Ojeda \& Winn 2011).

The presence of a massive close-in planet may induce tides that can lead to multiperiodic non-radial pulsations and in special cases radial pulsations of the host star (Schuh 2010; Herrero et al. 2011). However, only a handful set of pulsating exoplanet hosts has been found so far. V391b, for example, has been found orbiting an sdB star through pulsation-timing variations (Silvotti et al. 2007). The first main-sequence star where asteroseismology was applied to exoplanetary research is $\mu$ Arae (Bazot et al. 2005). High-precision and long-term Kepler observations have led to the discovery of several transiting exoplanet host stars which exhibited solar-like oscillations (Davies et al. 2016), whose typical semi-amplitudes are of the order of a few ppm for main-sequence stars (Baudin et al. 2011).

WASP-33 was the first transiting-exoplanet host star exhibiting $\delta$ Scuti pulsations, with a semi-amplitude of about $900 \mathrm{ppm}$ (Collier Cameron et al. 2010; Herrero et al. 2011). One of the harmonics has 
a frequency 26 times the orbital frequency, suggesting that WASP33 's pulsations might be induced by the planet. A more confident claim for the planet-induced stellar pulsations was made recently for HAT-P-2 system, a possible low-amplitude $\delta$ Scuti pulsator with an 87-min pulsation period and an amplitude of $40 \mathrm{ppm}$ (de Wit et al. 2017). Its pulsation modes correspond to exact harmonics of the planet's orbital frequency and are thought to be induced by the transient tidal interactions with its massive $\left(8 M_{\text {Jup }}\right)$, short-period (5.6 d) and highly eccentric $(e=0.5)$ planet (de Wit et al. 2017). Systems such as WASP-33 and HAT-P-2 provide a laboratory to study star-planet interactions. The observations provided by the $K 2$ mission have led to the discovery of another pulsating transiting exoplanet host star, namely HAT-P-56, which is likely a $\gamma$ Doradus pulsator (Huang et al. 2015). While the exact asteroseismic analysis approach can vary depending on the class of variability, one can investigate any potential star-planet interactions and derive precise stellar mass, radius and the depth-dependent chemical composition, given the appropriate modelling capabilities (Schuh 2010).

In this paper, we present a detection of starspots in the $K 2$ light curve of WASP-107 and pulsations in the light curve of WASP118. We also refine system parameters for both systems, search for transit-timing variations (TTVs), phase-curve modulations and any additional transiting planets and provide a measurement of the rotational period for WASP-107.

Simultaneously with our paper, Dai \& Winn (2017) have announced an analysis of the same $K 2$ short-cadence observations of WASP-107. Their results are in good agreement with ours.

\section{THE K2 OBSERVATIONS}

WASP-107 was observed by $K 2$ in the 1 -min short-cadence observing mode during Campaign 10 between 2016 July 6 and 2016 September 20. The dominant systematics present in the $K 2$ light curves are the sawtooth-shaped artefacts caused by the drift of the spacecraft. We attempted to correct for the drift artefacts using the same self-flat-fielding (SFF) procedure as described in Močnik et al. (2016b). However, since the drifts of the spacecraft were variable during this observing campaign, we obtained better results using the K2 Systematic Correction (K2SC) pipeline (Aigrain, Parviainen $\&$ Pope 2016), which uses break points to isolate different drift behaviours to overcome the issue of inconsistent drifts. The K2SC procedure was designed for the $K 2$ long-cadence observing mode and had to be modified slightly to accept the short-cadence data. The main modification was to split the input short-cadence light curve into smaller overlapping sections, which were processed individually by K2SC and then merged back together using the overlapping sections. However, since the WASP-107 light curve exhibits pronounced modulations, the K2SC failed to correct the drift artefacts properly at three different 2-d-long sections centred at BJD 2457624.7, 2457631.8 and 2457638.3. We replaced these sections with the light curve we obtained using the above-mentioned SFF procedure. Using this approach, we achieved a median 1-min photometric precision of $260 \mathrm{ppm}$, compared to $870 \mathrm{ppm}$ before the artefact correction and $300 \mathrm{ppm}$ using only the SFF procedure. The final drift-corrected light curve of WASP-107 is shown in Fig. 1. We used this light curve to study the rotational modulation of the host star in Section 6. For all other analyses we used the normalized version of the light curve, which we produced with PYKE tool KEPFLATTEN (Still \& Barclay 2012), which divides the measured flux with a low-order polynomial fit with window and step sizes of 3 and $0.3 \mathrm{~d}$, respectively, which effectively removed any low-frequency modulations.

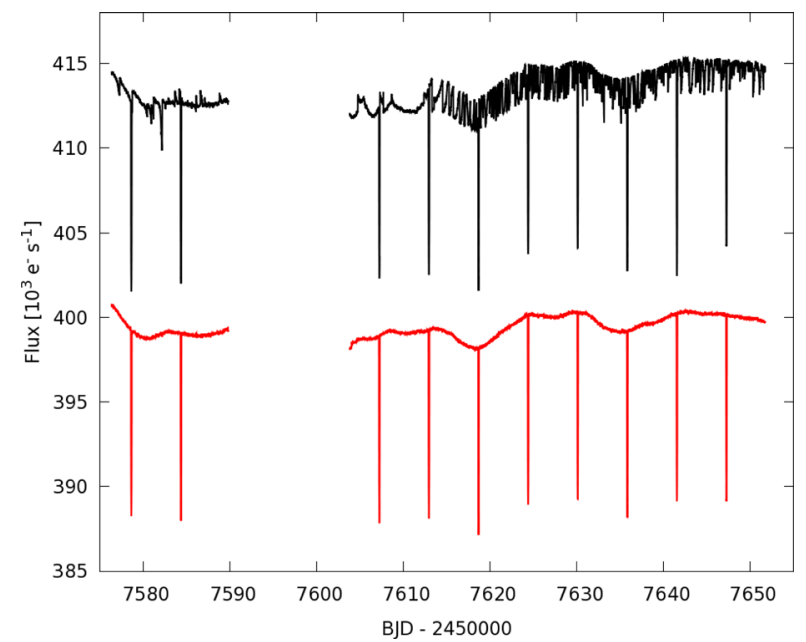

Figure 1. The binned light curve of WASP-107 shown before (in black) and after the drift correction (red). A 10-min binning was used to reduce the white noise and to display the drift artefacts more clearly. Note the presence of 10 transits and a rotational modulation. The 14-d data gap in the first half of the observing campaign was caused by a failure of CCD module 4 , which temporarily powered off the entire photometer. The corrected light curve is shown with an offset of $-14000 \mathrm{e}^{-} \mathrm{s}^{-1}$ for clarity.

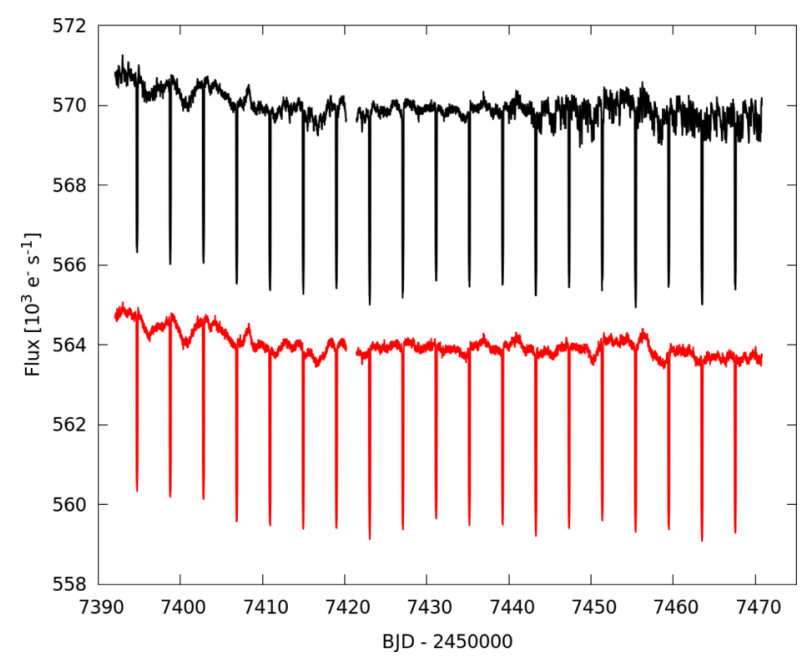

Figure 2. The binned light curve of WASP-118 shown before (in black) and after the drift correction (red). As in Fig. 1, we applied a 10-min binning to reduce the white noise. 19 transits are visible along with other variability. The corrected light curve is shown with an offset of $-6000 \mathrm{e}^{-} \mathrm{s}^{-1}$.

WASP-118 was observed during the $K 2$ Campaign 8 between 2016 January 4 and 2016 March 23, also in the short-cadence mode. Because the spacecraft's drifts were more consistent during Campaign 8 , we produced a slightly better light curve with our SFF procedure than with K2SC. The drift-corrected light curve of WASP118 is shown in red in Fig. 2. In addition to the transits, the light curve exhibits variability with an $\sim 5$-d time-scale. The variability is inconsistent and incoherent, and cannot be interpreted as a rotational modulation with confidence. We produced the normalized version of the light curve in the same way as for WASP-107. The normalized light curve revealed multiperiodic higher-frequency variability with a semi-amplitude of $\sim 200 \mathrm{ppm}$ (see Fig. 3). We suggest that this variability is produced by the weakly pulsating host star (see Section 7). 

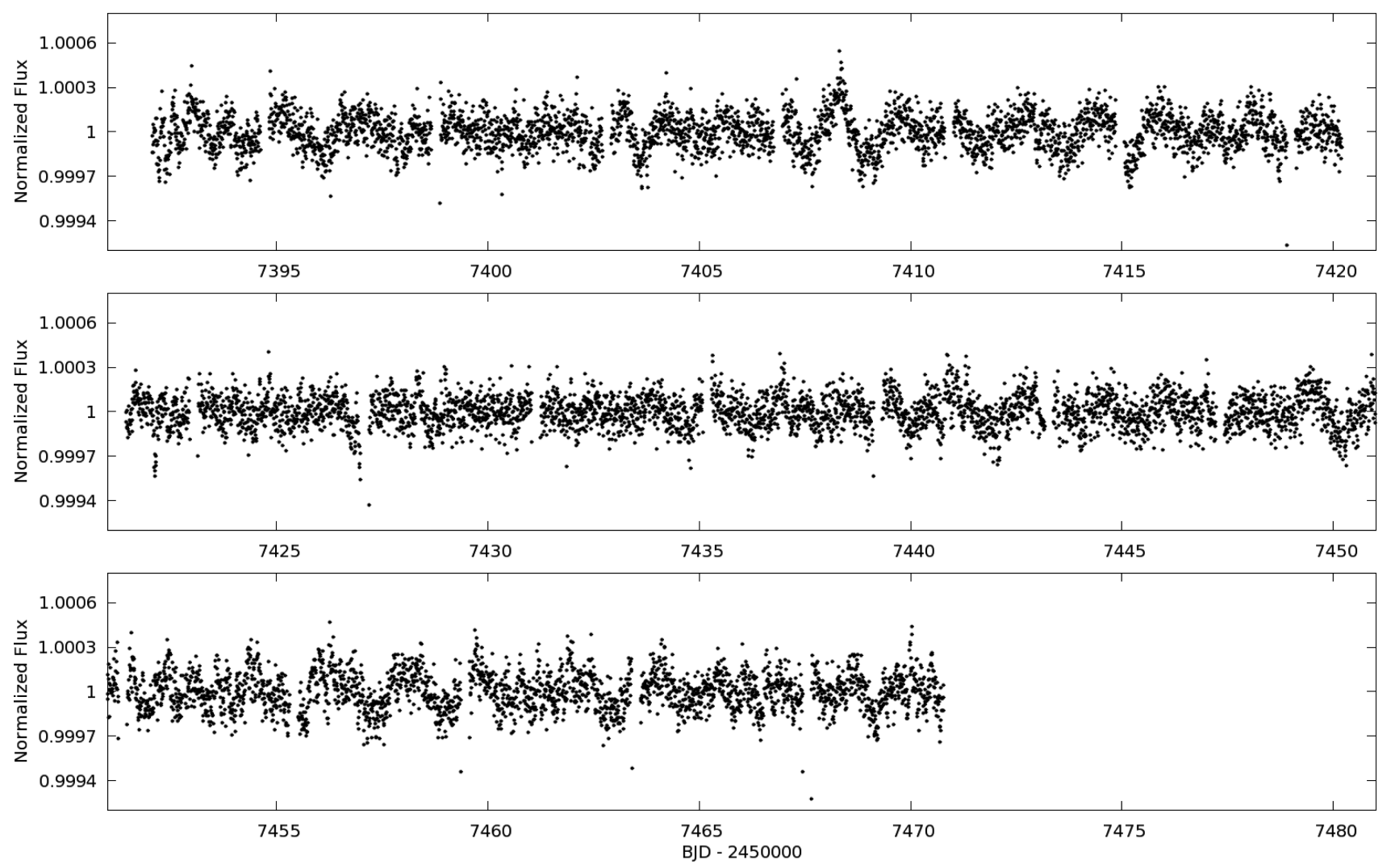

Figure 3. Stellar variability of WASP-118 resembling weak $\gamma$ Doradus pulsations. We show the normalized light curve with 10-min binning.

Because the presence of pulsations in the light curve of WASP118 could affect the analysis, we used the light curve with embedded pulsations only for the pulsations analysis in Section 7. For every other aspect of analysis we produced another light curve in which we removed these higher-frequency, low-amplitude pulsations. This was done by modifying our SFF procedure. Instead of the usual 5-d time-steps, we split the light curve into sections of individual spacecraft drifts before applying the SFF correction. Since the typical 6-h time-scale at which the drifts occur is considerably shorter than the observed pulsation time-scales, our modified SFF procedure effectively removed the pulsations from the light curve, along with any other variabilities at longer time-scales. This produced the light curve with a final median 1-min photometric precision of $210 \mathrm{ppm}$. We used this version of light curve for every aspect of the analysis, except for the analysis of pulsations in Section 7.

\section{SYSTEM PARAMETERS}

To determine the system parameters, we simultaneously analysed the $K 2$ transit photometry and radial-velocity measurements for both planets using a Markov Chain Monte Carlo (MCMC) code (Collier Cameron et al. 2007; Pollacco et al. 2008; Anderson et al. 2015). We used all the radial-velocity data sets that have been reported in the corresponding discovery papers (Anderson et al. 2017 and Hay et al. 2016), except the HARPS-N in-transit data set for WASP-118. This data set was excluded to prevent biasing the system parameters owing to a large scatter and underestimated error bars, as pointed out by the authors of the discovery paper. It may be that some of this scatter results from the pulsations that we now report. Limb darkening was accounted for using a four-parameter law, with co- efficients calculated for the Kepler bandpass and interpolated from the tabulations of Sing (2010).

For the MCMC analysis of WASP-107 we used the normalized light curve, from which we excluded any starspot occultation events (see Section 5), because failing to do so may lead to inaccurate system parameter determination, as discussed by Oshagh et al. (2013). Similarly, the presence of pulsations in the light curve of WASP118 may have affected the precise transit analysis and so we instead used the light curve with pulsations removed.

We first imposed a circular orbit in the main MCMC analysis for both planets. We then estimated the upper limits on eccentricities in a separate MCMC run by allowing the eccentricities to be fitted as a free parameter. To improve the precision of the orbital ephemerides, we also ran another MCMC analysis for both systems that included all the available ground-based photometric data sets used in the discovery papers which expanded the observational time-span and reduced the uncertainty on the orbital period by factors of 6.5 for WASP-107 and 2.3 for WASP-118. For these additional photometric data sets, we used limb-darkening coefficients from Claret (2000, 2004), as appropriate for different bandpasses.

The resulting system parameters are given in Table 1 and the corresponding transit models are shown in Figs 4 and 5.

\section{NO TTV OR TDV}

Inter-planet gravitational interactions can cause TTVs and transitduration variations (TDVs) (Agol et al. 2005). The detection of these variations can therefore reveal additional planets in the system. Typical reported TTV amplitudes range from a few seconds and up to several hours with periods of the order of a few days (Mazeh 
Table 1. MCMC system parameters for WASP-107 and WASP-118.

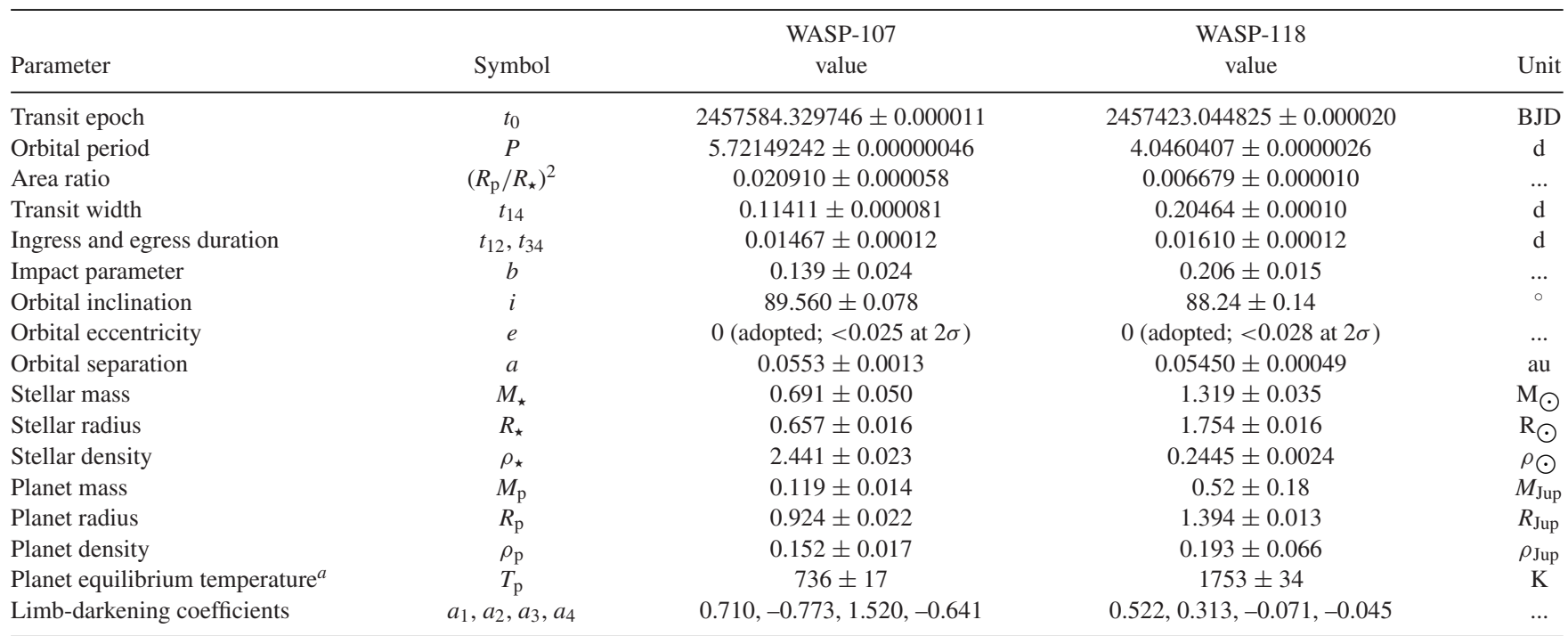

Note. ${ }^{a}$ Planet equilibrium temperature is based on assumptions of zero Bond albedo and complete heat redistribution.

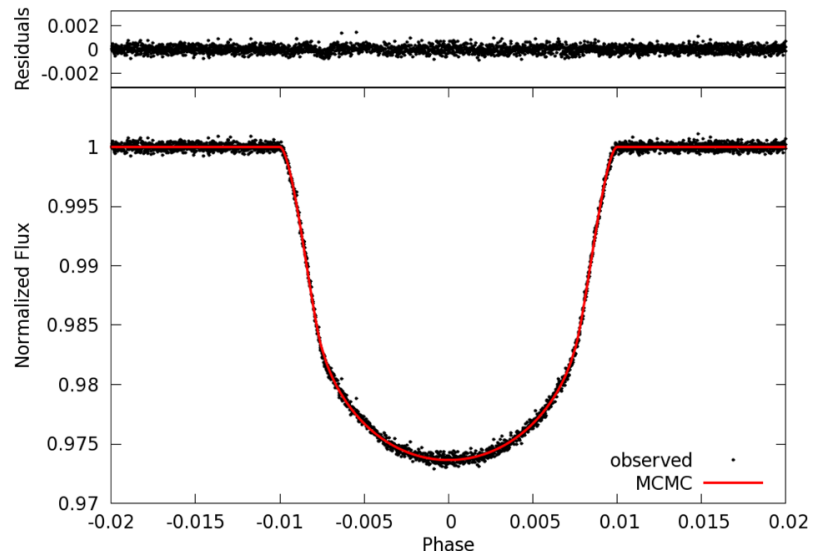

Figure 4. Best-fitting MCMC transit model and its residuals for WASP107.

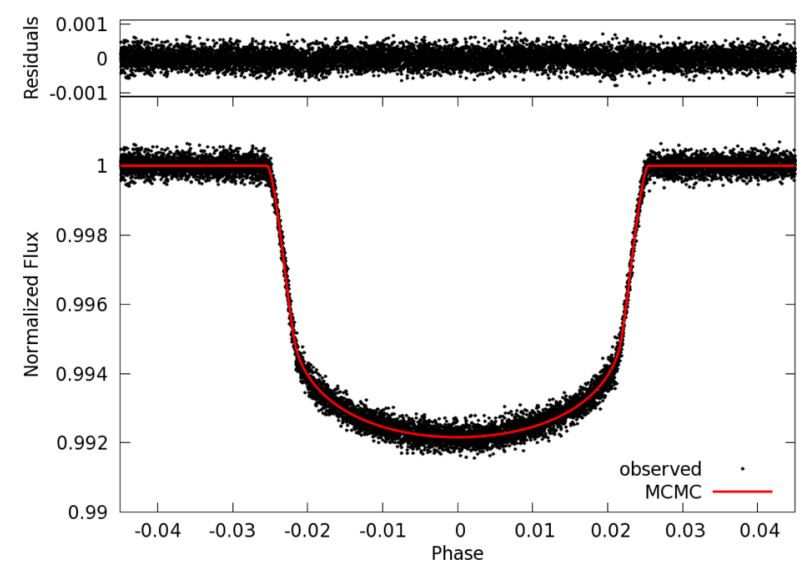

Figure 5. Best-fitting MCMC transit model and its residuals for WASP118.

et al. 2013). TDVs are expected to be in phase with the TTVs but at a significantly lower amplitude (Nesvorný et al. 2013).

To search for TTVs and TDVs, we ran another MCMC analysis on individual transits for both systems. We again removed the starspot occultation events from the WASP-107 light curve and used the pulsation-free light curve of WASP-118 since the light curve variability could affect the timing accuracy (Oshagh et al. 2013).

Against the hypothesis of equal transit-timing spacings and constant transit durations, the measured TTVs and TDVs for WASP-107 correspond to the $\chi^{2}$ values of 11.1 and 6.6, respectively, for 10 degrees of freedom. Similarly, for WASP-118 the TTV and TDV $\chi^{2}$ values are 26.8 and 15.7, respectively, for 19 degrees of freedom. Thus there are no significant TTVs or TDVs. The upper limits for WASP-107 are 20 and $60 \mathrm{~s}$ for TTVs and TDVs, respectively, for periods shorter than $80 \mathrm{~d}$. For WASP-118 the upper limits are 40 and $100 \mathrm{~s}$. Given the absence of any statistically significant TTV or TDV variations, we can conclude that any additional close-in, massive planets are unlikely in either of the two systems.

\section{STARSPOTS ON WASP-107}

In Fig. 6, we show the light curve of WASP-107 centred at individual transits after subtracting the best-fitting MCMC transit model from Section 3. The residual light curve reveals several starspot occultation events. Each of us has individually examined the residual light curve by eye and marked the events as definite or possible occultations. Here we report occultation events that were marked by at least two colleagues. This gives five definite starspot occultation events (marked with dark-red ellipses in Fig. 6) and four possible events (marked with light-red ellipses) within the 10 observed transits. Table 2 lists all the marked definite and possible occultation events, measured orbital phases at which they occur and the corresponding stellar longitudes which we calculated using the system parameters from Table 1 . There may well be additional, smalleramplitude spots present in some of the transits, in addition to those listed.

Given the stellar rotational period of $17.5 \pm 1.4 \mathrm{~d}$ (see Section 6) and the planet's orbital period of $5.72 \mathrm{~d}$ (see Table 1), one would expect in an aligned system to see the same starspot being occulted again three transits later, with a longitude shift of $-7_{-26}^{\circ+31}$. Occultations one, two or four transits later would be hard to detect, since the phase shifts would be in multiples of $120^{\circ}$, and therefore the 


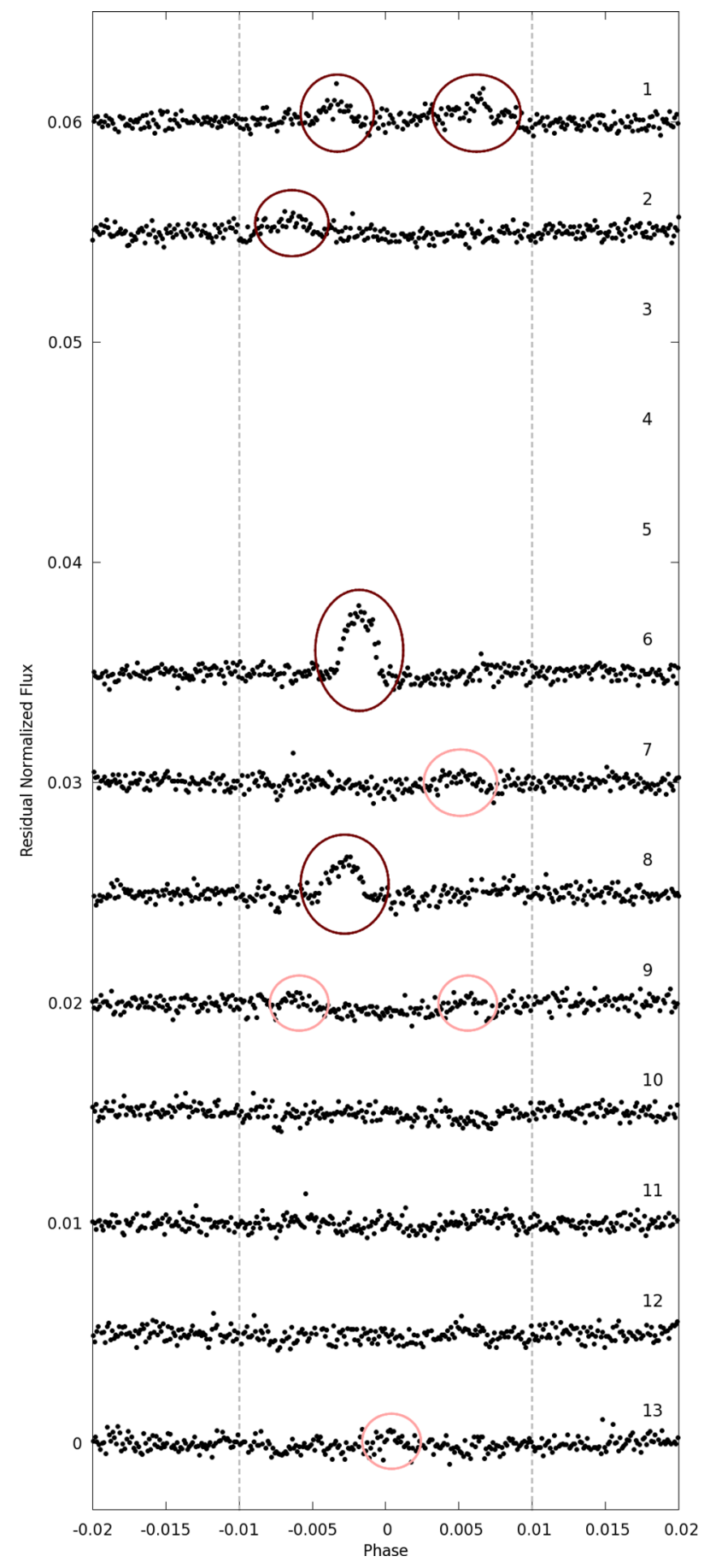

Figure 6. Starspot occultations in the model-subtracted light curve of WASP-107. Vertical dashed lines show the extent of the transit. Dark-red ellipses mark definite starspot occultation events and light-red ellipses mark possible starspots. Transits 3-5 were not observed owing to the 14-d data gap.

spots would either be close to the limb, where they are hard to detect owing to limb darkening, or not on the visible face at all.

There is only one pair of starspot occultations that might be a recurrence. If the occultation event in transit 6 and the first occultation in transit 9 (see Fig. 6) were caused by the same starspot, they would imply an orbital period of $18.8 \pm 0.2 \mathrm{~d}$. Although this is compatible with the $17.5 \pm 1.4-\mathrm{d}$ rotational period derived from the rotational modulation (see Section 6), there are reasons to doubt that the pair
Table 2. Phase and longitude positions of every detected starspot occultation event.

\begin{tabular}{lrr}
\hline $\begin{array}{l}\text { Transit } \\
\text { number }\end{array}$ & Phase & \multicolumn{1}{c}{$\begin{array}{c}\text { Stellar } \\
\text { longitude }\end{array}$} \\
\hline 1 & $\left.{ }^{\circ}\right)$ \\
1 & $-0.0033 \pm 0.0002$ & $-22.4 \pm 1.6$ \\
2 & $0.0062 \pm 0.0002$ & $45.0 \pm 2.2$ \\
6 & $-0.0064 \pm 0.0002$ & $-47.6 \pm 2.4$ \\
7 & $-0.0018 \pm 0.0002$ & $-12.1 \pm 1.4$ \\
8 & $0.0051 \pm 0.0002$ & $36.2 \pm 1.9$ \\
9 & $-0.0028 \pm 0.0002$ & $-18.5 \pm 1.5$ \\
9 & $-0.0059 \pm 0.0002$ & $-42.9 \pm 2.1$ \\
13 & $0.0056 \pm 0.0002$ & $40.3 \pm 2.0$ \\
\hline
\end{tabular}

Note. ${ }^{a}$ Longitude runs from $-90^{\circ}$ (first planetary contact), through $0^{\circ}$ (central meridian) to $90^{\circ}$ (last contact).

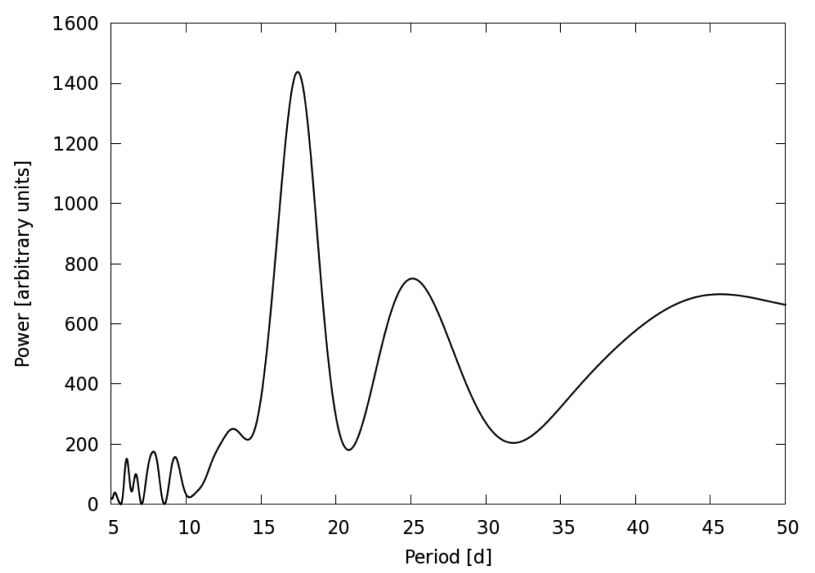

Figure 7. Lomb-Scargle periodogram of WASP-107's rotational modulation. The highest peak implies a rotational period of $17.5 \pm 1.4 \mathrm{~d}$.

was actually caused by the same starspot. First, a starspot from transit 8 does not produce an occultation pair at a similar phase shift in transit 11. Secondly, the starspot lifetimes for main-sequence stars are of the order of days (Bradshaw \& Hartigan 2014 and citations therein), which means that in $17 \mathrm{~d}$, the time it takes for the planet to orbit its host star three times, a starspot could disappear.

Overall, we do not find compelling evidence for recurring starspots, which would suggest that the system might be misaligned. However, because of the 17-d time span between recurrences that could be readily observed, we cannot exclude the possibility that it is an aligned system with relatively short starspot lifetimes.

\section{ROTATIONAL MODULATION OF WASP-107}

The light curve of WASP-107 in Fig. 1 reveals a low-frequency modulation with a semi-amplitude of about 0.2 per cent. To measure the periodicity of this modulation, we removed the planetary transits and calculated a Lomb-Scargle periodogram (see Fig. 7). The highest peak in the periodogram and its full width at half-maximum corresponds to a periodicity of $17.5 \pm 1.4 \mathrm{~d}$.

Knowing that the stellar surface harbours starspots (see Section 5), we believe that this modulation is caused by the stellar rotation. Using the stellar radius from Table 1 and assuming that the rotational axis is orthogonal to the line of sight, the measured rotational modulation period implies a stellar rotational velocity of $1.9 \pm 0.2 \mathrm{~km} \mathrm{~s}^{-1}$. This value agrees with the spectroscopic projected rotational velocity of $2.5 \pm 0.8 \mathrm{~km} \mathrm{~s}^{-1}$ (Anderson et al. 2017). Our 


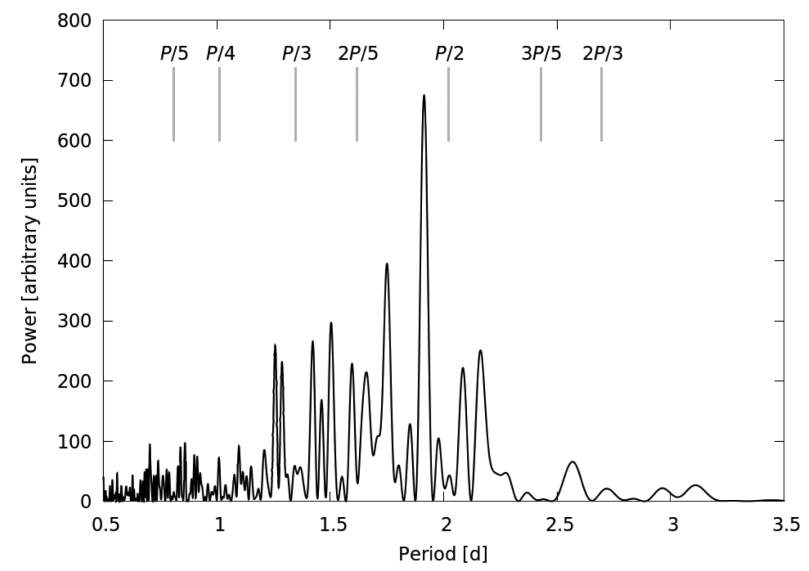

Figure 8. The Lomb-Scargle periodogram of WASP-118's higherfrequency variability in the normalized version of the light curve. Several peaks between 1 and $2.5 \mathrm{~d}$ indicate that the higher-frequency variability is multiperiodic. Also shown are some of the main harmonics of the planet's orbital period which demonstrate a mismatch with the pulsation periodicities. Note that we show here only a few of the main harmonics for clarity. The power spectral density for periods longer than $3.5 \mathrm{~d}$ is virtually zero because of the applied flattening normalization procedure.

rotational modulation period and amplitude also agree well with the period of $17 \pm 1 \mathrm{~d}$ and amplitude of 0.4 per cent that were derived from the ground-based photometry and reported in the discovery paper by Anderson et al. (2017).

\section{STELLAR PULSATIONS OF WASP-118}

Fig. 3 provides a close-up view of the higher-frequency photometric variability in the light curve of WASP-118, after removing incoherent low-frequency modulations. This variability is not correlated with the spacecraft's drifts, is preserved using four different datareduction procedures, is not present in the $K 2$ light curves of other nearby stars and is not correlated with the orbital phase.

The variability cannot be realistically considered as a rotational modulation because its short 2-d period would require the star to rotate three times faster than the spectroscopically measured projected rotational velocity. This would be possible only if the system was hugely misaligned, in contradiction with the Rossiter-McLaughlin measurements by Hay et al. (2016), who suggested that the system is aligned. The variability is therefore most likely to be weak pulsations of the host star.

The semi-amplitude of the pulsations is $\sim 200 \mathrm{ppm}$. The LombScargle periodogram reveals that the variability is multiperiodic (see Fig. 8) with the highest peak at 1.9 d. To check whether the normalization procedure affected the detected pulsations, we ran signal injection tests and found that the applied normalization procedure preserves 80 per cent of the variability at the peak pulsation period near $1.9 \mathrm{~d}$.

Kaye et al. (1999) introduced a $\gamma$ Doradus pulsating type for main-sequence and subgiant stars of spectral types A7-F5. $\gamma$ Doradus stars exhibit non-radial, high-order and low-degree multiperiodic pulsations with periods of $0.4-3 \mathrm{~d}$ and amplitudes below $0.1 \mathrm{mag}$. The pulsation amplitude of such stars may vary during an observing season by as many as a factor of 4 .

The spectral type and variability characteristics of WASP-118 suggest that the star is probably a weak, late-type $\gamma$ Doradus pulsator.
If the stellar pulsations were induced by the star-planet interactions, we would expect them to appear at exact harmonics of the planet's orbital period, such as in the cases of WASP-33 (Herrero et al. 2011) and HAT-P-2 (de Wit et al. 2017). We found no match when comparing the main harmonics of the WASP-118b's orbital period with the measured pulsations' periodicities (see Fig. 8). Despite the lack of any commensurabilities with the main harmonics, we cannot yet reject any complex commensurabilities nor the possibility that the pulsations were induced by the orbiting planet. A detailed follow-up asteroseismic analysis is required to further investigate the star-planet interactions as a possible cause for the observed pulsations in the light curve of WASP-118.

\section{NO PHASE-CURVE MODULATIONS}

Phase curves in exoplanet systems consist of three main components at optical wavelengths: (1) ellipsoidal modulation, (2) Doppler beaming and (3) planetary reflection (e.g. Esteves, De Mooij \& Jayawardhana 2013). Additionally, a transiting planet may produce a secondary eclipse, an occultation of the planet by its host star, which blocks the reflected light during the occultation. Therefore, the depth of the secondary eclipse is twice the semi-amplitude of the reflectional modulation.

To produce a phase curve of WASP-107, we had to remove the significant rotational modulation prior to phase-folding the light curve. However, because the ratio between the 5.7-d orbital period and the 17-d rotational modulation is not small enough, this would also remove any phase-curve modulations. For WASP-118 we again removed stellar variability, which would again remove any phasecurve modulations. However, in both systems the procedures would not have removed any secondary eclipses present. A non-detection of secondary eclipses in both systems therefore allowed us to estimate conservative upper limits on the secondary-eclipse depths of 100 ppm for WASP-107 and 50 ppm for WASP-118.

Using the system parameters from Table 1, the theoretically expected semi-amplitudes of ellipsoidal, Doppler beaming and reflectional modulation for WASP-107 are $0.03,0.2$ and $75 A_{\mathrm{g}} \mathrm{ppm}$, respectively, where $A_{\mathrm{g}}$ is the planet's geometrical albedo. For WASP118 , the expected semi-amplitudes are $1,0.7$ and $150 A_{\mathrm{g}} \mathrm{ppm}$. These amplitudes have been calculated using the relations from Mazeh \& Faigler (2010). While the expected amplitudes for the ellipsoidal and Doppler beaming modulations are below the $K 2$ photometric precision, the inflated planetary radii could produce a significant reflectional modulation in both systems. Using the upper limits for secondary eclipse depths and theoretically expected amplitudes for reflectional modulations allows us to constrain the planetary geometric albedos to less than 0.7 for WASP-107b and less than 0.2 for WASP-118b.

\section{NO ADDITIONAL TRANSITING PLANETS}

To search for signatures of any additional transiting planets in the normalized light curves of both systems, we first removed the transits of the known transiting planets and then calculated the boxleast-square periodograms of any other periodic signals with the PYKE tool KEPBLS. The absence of any significant residual signals in the period range $0.5-30 \mathrm{~d}$ results in transit-depth upper limits of $130 \mathrm{ppm}$ for any additional transits in the WASP-107 system and 140 ppm in WASP-118. 


\section{AGES OF THE HOST STARS}

We estimated the ages of both host stars by comparing the measured stellar densities from Table 1 and the published spectroscopic effective temperatures to isochrones computed from the stellar evolution models. This was done with the Bayesian mass and age estimator Bagemass (Maxted, Serenelli \& Southworth 2015a), which uses the GARSTEC code (Weiss \& Schlattl 2008) to compute the evolution models. The best-fitting stellar evolution tracks provided the age estimates of $8.3 \pm 4.3$ Gyr for WASP-107 and $2.3 \pm 0.5$ Gyr for WASP-118.

The rate at which a star rotates acts as another age estimator. Over time, stars lose angular momentum through magnetised stellar winds and gradually slow down (Barnes 2003). Knowing the rotational period of WASP-107 from the detected rotational modulation (see Section 6), we estimated the stellar age with the gyrochronological relation by Barnes (2007) to obtain $0.6 \pm 0.2 \mathrm{Gyr}$.

Our isochronal age estimate for WASP-118 agrees well with the age provided by Hay et al. (2016), who used the same approach but using the system parameters derived only from the ground-based observations.

The age discrepancy between the isochronal and gyrochronological age estimate for WASP-107 is significant. Similar discrepancies have been observed for many other K-type stars hosting transiting exoplanets (e.g. Maxted, Serenelli \& Southworth 2015b). It has been suggested that stars hosting massive short-period planets may have been spun up by the tidal interaction with the planet and thus exhibit a lower gyrochronological age (Maxted et al. 2015b). However, the warm-Saturn WASP-107b is not massive enough and does not orbit close enough to its host star to cause a significant tidal spin-up. A more probable reason for the observed age discrepancy in the case of WASP-107 is the radius anomaly, in which latetype stars exhibit larger radii than is predicted by the stellar models (Popper 1997). The radius anomaly is an active research topic driven by the advances in simulating convections in low-mass stars (Ludwig, Caffau \& Kučinskas 2008) and incorporating magnetic fields into stellar models (Feiden \& Chaboyer 2013). Morales et al. (2010) have demonstrated that the presence of starspots near the poles of low-mass stars could affect the stellar radii and cause the observed radius anomaly. The age discrepancy of magnetically active K-type WASP-107 may therefore be tentatively attributed to starspots.

\section{CONCLUSIONS}

The two main results presented in this paper are the direct detection of magnetic activity in the short-cadence $K 2$ light curve of WASP107 and the detection of stellar variability of WASP-118.

The magnetic activity of WASP-107 is manifest first as a rotational modulation which gives a stellar rotational period of $17.5 \pm 1.4 \mathrm{~d}$. We also detect a total of five definite and four possible starspot occultation events. With the planet's orbital period being nearly one-third of the rotational period of the star, we might expect to see the same starspot recurring every three transits. Since we found no evidence of recurring starspots, we suggest that the system is misaligned, unless the starspots' lifetimes are shorter than the rotational period of the star.

The multiperiodic variability in the light curve of WASP-118 indicates that the star is likely a low-amplitude $\gamma$ Doradus pulsator. WASP-118 is a good target for a follow-up asteroseismic analysis in order to obtain more precise stellar parameters and to investigate star-planet interactions as a possible cause for the observed stellar pulsations.
Our refinement of WASP-107 system parameters may also prove beneficial, since the planet lies in the transition region between ice and gas giants. Knowing precise system parameters of such planets is crucial for understanding why some ice giants do not become gas giants.

\section{ACKNOWLEDGEMENTS}

We thank the anonymous referee for their helpful comments. We also thank Prof. Suzanne Aigrain for her help modifying the K2SC data reduction procedure to accept the $K 2$ short-cadence data. We gratefully acknowledge the financial support from the Science and Technology Facilities Council, under grants ST/J001384/1, ST/M001040/1 and ST/M50354X/1. This paper includes data collected by the $K 2$ mission. Funding for the $K 2$ mission is provided by the NASA Science Mission directorate. This work made use of PYKE (Still \& Barclay 2012), a software package for the reduction and analysis of Kepler data. This open source software project is developed and distributed by the NASA Kepler Guest Observer Office. This research has made use of the NASA Exoplanet Archive, which is operated by the California Institute of Technology, under contract with the National Aeronautics and Space Administration under the Exoplanet Exploration Program.

\section{REFERENCES}

Agol E., Steffen J., Sari R., Clarkson W., 2005, MNRAS, 359, 567 Aigrain S., Parviainen H., Pope B. J. S., 2016, MNRAS, 459, 2408 Anderson D. R. et al., 2015, A\&A, 575, A61

Anderson D. R. et al., 2017, A\&A, preprint (arXiv:1701.03776)

Barnes S. A., 2003, ApJ, 586, 464

Barnes S. A., 2007, ApJ, 669, 1167

Baudin F. et al., 2011, A\&A, 529, A84

Bazot M., Vauclair S., Bouchy F., Santos N. C., 2005, A\&A, 440, 615

Borucki W. J. et al., 2010, Science, 327, 977

Bradshaw S. J., Hartigan P., 2014, ApJ, 795, 79

Claret A., 2000, A\&A, 363, 1081

Claret A., 2004, A\&A, 428, 1001

Collier Cameron A. et al., 2007, MNRAS, 380, 1230

Collier Cameron A. et al., 2010, MNRAS, 407, 507

Dai F., Winn J. N., 2017, AJ, 153, 205

Dai F., Winn J. N., Yu L., Albrecht S., 2017, AJ, 153, 40

Davies G. R. et al., 2016, MNRAS, 456, 2183

de Wit J. et al., 2017, ApJ, 836, L17

Esteves L. J., De Mooij E. J. W., Jayawardhana R., 2013, ApJ, 772, 51

Feiden G. A., Chaboyer B., 2013, ApJ, 779, 183

Hay K. L. et al., 2016, MNRAS, 463, 3276

Herrero E., Morales J. C., Ribas I., Naves R., 2011, A\&A, 526, L10

Howell S. B. et al., 2014, PASP, 126, 398

Huang C. X. et al., 2015, AJ, 150, 85

Kaye A. B., Handler G., Krisciunas K., Poretti E., Zerbi F. M., 1999, PASP, 111,840

Ludwig H.-G., Caffau E., Kučinskas A., 2008, in Deng L., Chan K. L., eds, IAU Symp. Vol. 252, The Art of Modelling Stars in the 21st Century. Cambridge Univ. Press, Cambridge, p. 75

Maxted P. F. L., Serenelli A. M., Southworth J., 2015a, A\&A, 575, A36

Maxted P. F. L., Serenelli A. M., Southworth J., 2015b, A\&A, 577, A90

Mazeh T., Faigler S., 2010, A\&A, 521, L59

Mazeh T. et al., 2013, ApJS, 208, 16

Morales J. C., Gallardo J., Ribas I., Jordi C., Baraffe I., Chabrier G., 2010, ApJ, 718, 502

Močnik T., Southworth J., Hellier C., 2016a, MNRAS, preprint (arXiv:1608.07524) 
Močnik T., Clark B. J. M., Anderson D. R., Hellier C., Brown D. J. A., 2016b, AJ, 151, 150

Nesvorný D., Kipping D., Terrell D., Hartman J., Bakos G. Á., Buchhave L. A., 2013, ApJ, 777, 3

Oshagh M., Santos N. C., Boisse I., Boué G., Montalto M., Dumusque X., Haghighipour N., 2013, A\&A, 556, A19

Pollacco D. L. et al., 2006, PASP, 118, 1407

Pollacco D. et al., 2008, MNRAS, 385, 1576

Popper D. M., 1997, AJ, 114, 1195

Sanchis-Ojeda R., Winn J. N., 2011, ApJ, 743, 61

Schuh S., 2010, Astron. Nachr., 331, 489
Silva A. V. R., 2003, ApJ, 585, L147

Silvotti R. et al., 2007, Nature, 449, 189

Sing D. K., 2010, A\&A, 510, A21

Still M., Barclay T., 2012, Astrophysics Source Code Library, record ascl:1208.004

Weiss A., Schlattl H., 2008, Ap\&SS, 316, 99

This paper has been typeset from a $\mathrm{T}_{\mathrm{E}} \mathrm{X} / \mathrm{L} \mathrm{T} \mathrm{T}_{\mathrm{E}} \mathrm{X}$ file prepared by the author. 